\title{
A Generic Cognitive Radio Based on Commodity Hardware
}

\author{
John Sydor, David Roberts, Bernard Doray and Amir Ghasemi \\ Communications Research Centre Canada \\ 3701 Carling Avenue, Ottawa, Ontario, Canada K2H 8S2. \\ \{john.sydor, david.roberts, bdoray, aghasemi\}@crc.gc.ca
}

\begin{abstract}
In this paper we describe the process that we undertook to build a configurable wireless platform that can be used to implement cognitive radio network (CRN) architectures. Consisting of a commodity IEEE $802.11 \mathrm{a} / \mathrm{b} / \mathrm{g}$ (WiFi) router at the physical (PHY) layer and RF signal processing and IP traffic shaping circuitry, the resultant hybrid terminal (called the WiFi_CR) becomes a building block that can implement cognitive femtocells, point to multipoint, mesh, and relay wireless networks. The IP addressable WiFi_CR terminals can sense their radio environment, schedule IEEE 802.11 packet transmissions in space and time, and select channel, modulation rates, and transmit power. Intelligent operation is undertaken by a cognitive network management system (CR_NMS) which controls a number of WiFi_CR terminals and solicits sensor information from them. The CR_NMS gathers the spectrum and sensed interference information and builds a memory map with such knowledge, thereby creating radio environment awareness for the CRN. Cognitive engines (the intelligent control algorithms) within the CR_NMS use radio environment knowledge and other information (such as spectrum policy) to give the system a capability to seek white space spectrum, avoid interference, identify primary users, and take on other tasks associated with cognitive radio (CR). Designed around ISM band operation, this generic terminal, with proper RF modifications, can work in the TV bands, 3.65-3.70 GHz, and up to $60 \mathrm{GHz}$.
\end{abstract}

Index Terms-cognitive radio; WiFi; IEEE $802.11 \mathrm{a} / \mathrm{b} / \mathrm{g}$.

\section{INTRODUCTION}

The definitions and operating principals of cognitive radio are complicated by the reality that different wireless applications require different cognitive capabilities. TV band cognitive radios, for example, have different requirements on intelligent sensing and coexistence compared to public safety band radios working in the $4.9 \mathrm{GHz}$ bands or the DARPA XG radios that work in military environments. To do meaningful implementation-oriented work in CR often requires a researcher to commit to a specific technology, often at great cost in terms of experimental equipment and hardware development. Moreover, the requirements governing such radios themselves change or are based on questionable simulation data; thus further complicating the design process. Having a working cognitive radio whose operation is analogous to these highly specialized (often unavailable) CR systems would do much to alleviate the problems that researchers and development engineers face.

Our work on Cognitive Radio at the Communications Research Centre (CRC) in Ottawa, Canada, is focused on the development of cognitive radio systems that analytical studies show as being capable of MIMO-like capacity gains or reusing spectrum [1-3]. We are also interested in testing coexistence and primary user detection strategies that, though confidently proposed in evolving standards such as the IEEE 802.16h and IEEE $802.22[4,5]$, have few tested results in support of them. To undertake such diverse investigations we required a generic cognitive radio that could be modified in the lab and field tested in realistic environments. Defining such a radio was the first step toward implementing it.

\section{Five Structural Elements of a CR}

When undertaking an examination of the extensive literature dealing with cognitive radio it becomes apparent that five elements are generally repeatedly discussed, and depending on the application or cognitive concepts being implemented, the mix and complexity of these vary accordingly. Typically, cognitive radios have: (a) a signal processing subsystem with configurable PHY layer characteristics; (b) a sensing system that is in part dependent on the PHY layer but embodies its own unique signal processing features; (c) a Medium Access Control (MAC) subsystem that supports the physical configuration and allows sensing information to flow to (d) an addressable, dynamic memory which is either created by collating the information derived from the sensing subsystem or is embedded as a set of rules or policies, and (e), a set of cognitive (decision making) engines that process information from the addressable memory or have direct connection to the sensing systems and have the ability to modify the performance of the physical structure, in a manner enhancing the operation of the CR.

This paper will discuss how we forged each of the above elements within the constraints of an off-the-shelf technology and made an experimenter-friendly cognitive radio platform which we now use for testing and development.

\section{DETAILS OF IMPLEMENTATION}

\section{A. IEEE 802.11 router-radio: a Configurable PHY}

Recently there has been some interest in developing reconfigurable wireless terminals using commodity hardware [6, 7]. Building upon such work, it became apparent to us that certain IEEE $802.11 \mathrm{a} / \mathrm{g}$ radios could satisfy some of the requirements identified above, especially those related to PHY layer control. 
Single-board computers customized for wireless router applications (referred to as "routers" hereafter) are supplied by a number of vendors and sold at low cost making them attractive to researchers and wireless implementers. The routers can be equipped with multiple radio cards thereby providing multiband operation at 2.4 and $5.8 \mathrm{GHz}$. They can be modified, with the addition of RF frequency translation subsystems, to work in the TV bands or at $60 \mathrm{GHz}$, bands of experimental interest to CRC. Madwifi [8] is a linux-based driver for Atheros 802.11 chipsets, initially released by Atheros and later enhanced by the Linux developers' community. This driver has the capability to configure the IEEE $802.11 \mathrm{a} / \mathrm{g}$ radio chipset, allowing it to work in a number of atypical wireless RLAN modes. Modifications to the Madwifi driver made it possible to implement interference monitoring and to rapidly change the transmit power, channel, and modulation rate of the radio. Certain IEEE 802.11 protocol features such as RTS/CTS, acknowledgement, contention window size, and Clear Channel Assessment (CCA) threshold could also be individually and independently set on the radios. The ability to adjust or disable such features was important to our CR design since in our applications the decision as to when (or when not) to transmit RF packets was to become a deterministic process under cognitive control, and a central requirement for our configurable PHY layer. Essentially, the pseudo-random and asynchronous exponential back-off algorithm, which is a core feature of the IEEE 802.11 Distributed Coordination Function (DCF) protocol, had to be either entirely disabled or heavily limited to meet our needs.

Configuration changes to the Madwifi router can be done remotely using IP communications. It is also possible to modify the driver software so that sensing messages are returned by IP to the cognitive engines in the CR_NMS. Having IP-based control and sensing is appealing as the cognitive engines would only require a TCP/IP protocol stack to support the remote radio control and sensing functions for the CRN. By doing this we would be implementing a 'cognitive control channel', thus sparing us the effort and expense of designing one.

For these reasons we opted to build our configurable PHY layer radio around the Madwifi router-radio. We called this terminal the WIFI_CR. With a number of software modifications to the Madwifi driver as well as creation of a hardwarebased RF 'control shell' around the radio device, we were able to circumvent the CSMA/CA issues. Additionally, we could be undertaking such modifications and still use the DCF protocol of the IEEE 802.11 standard. DCF mediates the handshaking necessary to maintain the single Access Point (Hub) to multiple Station (Client terminal) hierarchy, with its registration protocols, encryption, broadcast beacon, and other functions necessary to the background configuration, maintenance, and operation of a WiFi network. Many of these functions would be reused in our CRN.

\section{B. Creating a Control Shell around the IEEE 802.11 Protocol}

Having made the choice to implement a hardware-based control shell around the router, we identified additional PHY functions needed for our development. These new functions, also controlled by IP messaging, would support a:

- system-wide synchronization and scheduling scheme to allow systemic monitoring and coordination of radio transmissions by the CRN;

- a spatial scheduling capability that would constrain the transmission and reception of packets to specific directions thereby giving the CRN the ability to spatially control interference and establish directional links.

- At a specific WIFI_CR, the retrieval of information about location, interference environment, propagation, other users' spectrum occupancy, and the terminal's own spectrum occupancy and throughput statistics.

All wireless networks designed for spectral efficiency are synchronous and have deterministic transmission. Standards that support cognitive radio (such as IEEE 802.22 and IEEE 802.16h) have such features and also use scheduled quiet periods during which coordinated sensing and interference quantification is undertaken. To be analogous to such standards, such features had to be available to our CR.

The ability to transmit on a packet-by-packet basis in a deterministic manner can be achieved in two ways with the WIFI_CR. If the router is configured to be an Access Point (AP), the CSMA/CA protocol can be completely disabled by manipulation of a hardware register, consequently two processes important to CSMA, the exponential backoff and CCA are not used. An Ethernet packet presented to the router will be transmitted with a minimum of delay, which is due only to the processing time of the router. However, such operation is not possible to program into the router if it is configured to be a Station (Client) device. To get around this, a FPGA-based Ethernet buffer board circuit (EBB) was developed and inserted between the router and its wireline backhaul connection (Fig. 1).

Composed of a dual port Ethernet transceiver and an Altera FPGA chip, the EBB receives, examines, and holds Ethernet packets in a FIFO while it mimics clear channel conditions to the router's radio. This is done by introducing a minimum noise floor to the radio's RF chain. Under such conditions the CSMA/CA (specifically CCA) process is forced to transmit an incoming Ethernet packet with a minimum amount of delay and in a repeatable deterministic manner. As soon as transmission is detected the EBB disengages the low noise source and proceeds to load the next packet into the router. This "one-shot" process continues as long as Ethernet packets are presented to the router. The FPGA also calculates the size of the Ethernet packet and determines the actual radio transmission duration for the packet.

By control of the transmission timing, we also achieve a de facto control of the reception timing. Whenever the router does not have a buffered Ethernet packet, it automatically enters the receive state. Thus, the router can be constrained to specific transmission and reception intervals, thus implementing Time Division Duplexing (TDD). To take full advantage of TDD, synchronization is required across the CRN. 


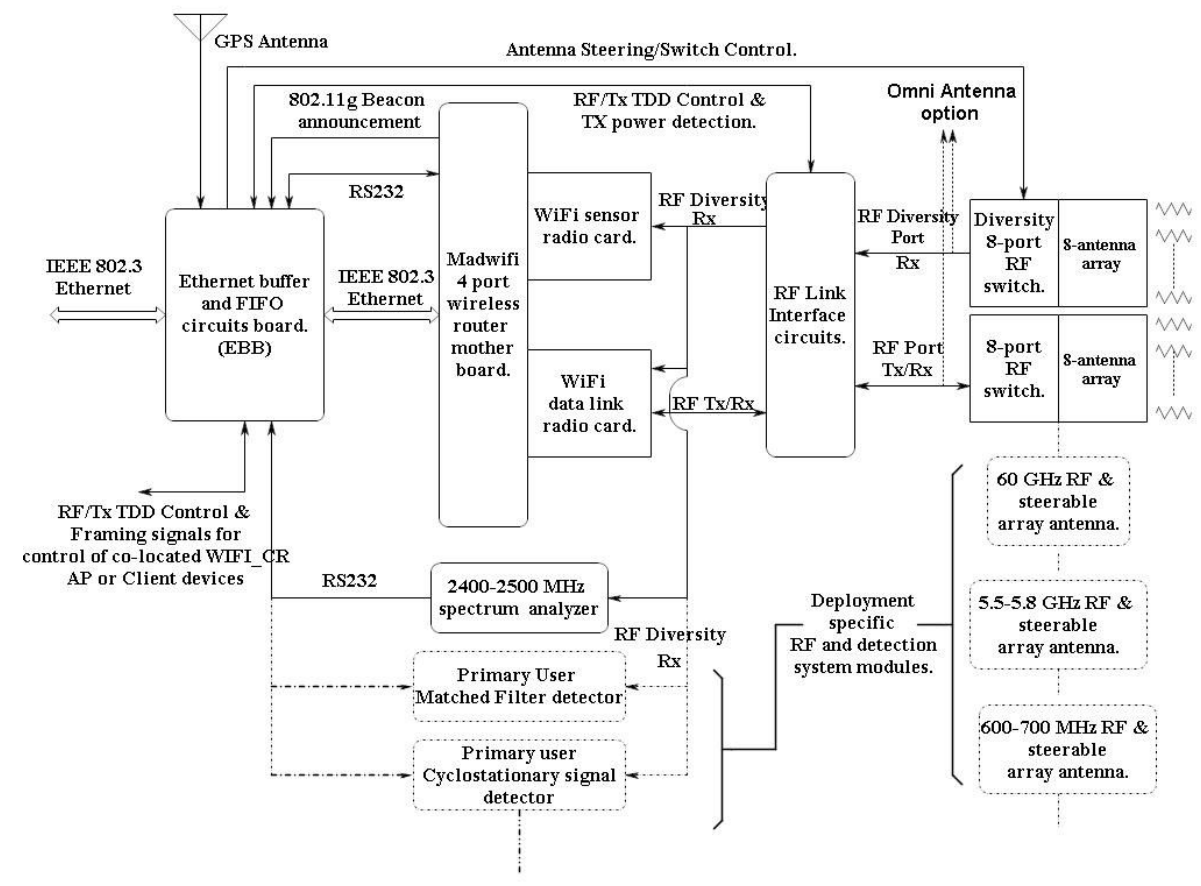

Fig. 1: WiFi_CR cognitive radio terminal PHY

\section{Implementing Synchronization}

WIFI_CR terminals use a repeating set of synchronized, numbered timeslots to coordinate transmission and reception periods. Timeslots are created by free-running counters in the FPGA that are clocked at $25 \mathrm{MHz}$ to $1 \mathrm{ppm}$ accuracy. The content of the counters is called Terminal Time (TT). For the WIFI_CR Timeslots are $4 \mathrm{msec}$ for link speed of $6 \mathrm{Mbps}$ and 2 msec for link speeds of 18 or 54 Mbps. TT can be reset by a synchronization pulse, generated in the following ways; options within the WIFI_CR:

- GPS Synchronization: a 1 PPS signal from the GPS resets TT once per second (+/- $15 \mathrm{nsec})$. This method is ideal for outdoor multi-hop networks, but does not work for indoor networks because of the GPS loss.

- Raw IEEE 802.11 Beacon Synchronization: this is the simplest method of synchronization, based on the detection of beacon signals transmitted by the AP as part of the DCF process of IEEE 802.11 standard. Reception of the beacon resets the TT and the network of AP and Client terminals re-synchronizes on each received one. If beacons are not received, transmission by a WIFI_CR is prevented (though reception is maintained). Beacons are the most resilient signals sent by the radio; Madwifi Client terminals undergo re-association with their AP if more than 11 consecutive beacons are missed, a standard practice in IEEE 802.11. Synchronization between AP and Client terminals is maintained within +/- 8 usecs. This method is useful for single-hop indoor CRNs.

- ARP-Assisted Synchronization: In this method when a beacon is generated by the AP router board, TT count at the moment of the beacon's end is broadcast to associated Clients via a modified TCP/IP Address Resolution Pro- tocol (ARP) packet; Clients continuously calculate the time since the received beacon ended, add this offset to the value received in the ARP message and then readjust their TT accordingly. Two such ARP packets are transmitted after each beacon for redundancy. This technique can be used to distribute synchronization in multihop links as each subsequent AP after the master AP operates at its own beacon period and the timeslot that it generates is corrected to that of the master AP. ARP-assisted synchronization can be used both outdoors and indoors, and can extend from outdoor APs into indoor APs. It can be beacon- or GPS-based (generated at the master AP) and be used for multiple hops. It is however, less resilient to propagation errors compared to simple beacon-based synchronization.

Multi-hop networks and relay nodes can be created by connecting Client and AP terminals back to back at their Ethernet ports; the WIFI_CR is capable of sharing a common timing in such collocated situations.

\section{PHY Layer Configurability of the EBB}

EBB's interfaces to attached devices (e.g. Router Board, Spectrum Analyzer board, GPS module, other WIFI_CR terminals) use common protocols (RS-232, RS-485, or Ethernet) and its code has been generalized as much as possible. Due to the flexibility of the code, it is possible to accommodate sensors (detailed below) customized for specific applications. Similarly, a flexible FIFO handling scheme is provided allowing the ability to allocate memory in support of either temporal or spatial packet scheduling within the WIFI_CR; however, due to the reprogrammable nature of the FPGA, it is possible to implement other modes for customized CR operation. 


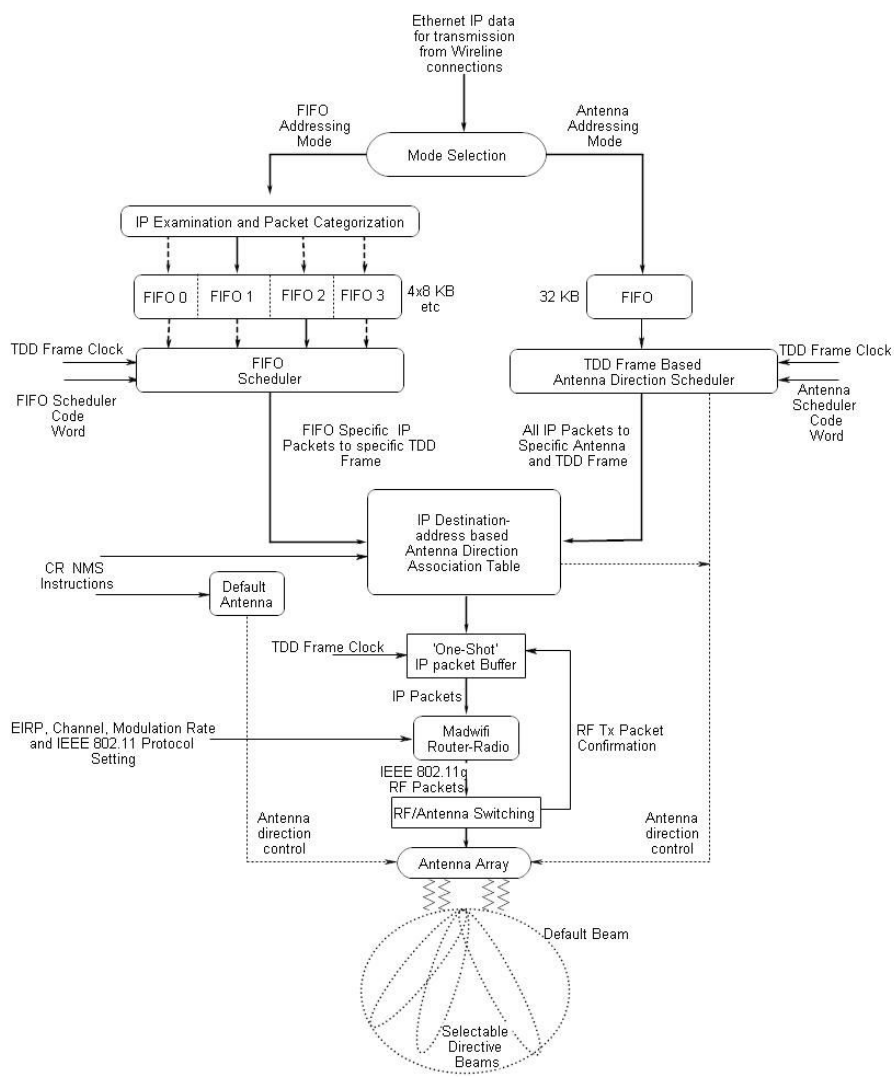

Fig. 2: FIFO-Centric and Antenna-Centric operation in the WiFi_CR

In the FIFO-centric mode (Fig. 2), the CR_NMS can both specify a table of destination IP addresses associated with each FIFO and the sequence as to which FIFO is to be emptied and transmitted in which timeslot. In this manner the cognitive engines can schedule interfering packets to interference free time slots. This technique for co-channel coexistence is analogous to that presented in the IEEE 802.16h amendment to the IEEE 802.16 (WiMAX) standard. Under this type of operation a default antenna (usually an omni-directional) is chosen and used throughout the WIFI_CR's operation.

Spatial control of packet transmissions is achieved using the Antenna-centric mode. In this mode all packets are held in a single FIFO and WIFI_CR specifies the direction of the transmissions for each time slot. This mode is useful for reconfigurable mesh networks and relay architectures.

The EBB is also capable of doing per-packet directional steering, a feature which can be implemented in the Antennacentric mode. The CR_NMS can allocate a specific Destination Address to an antenna direction, which is maintained for the duration in which the packet is actually transmitted until its IEEE 802.11 ACK is received. Packet steering is intended as a means of interference reduction and link budget enhancement, and can be used as a means of tracking and communicating with a mobile. Currently the WIFI_CR can select up to 8 antenna positions. This will be increased with the future versions of the terminal.

\section{E. Spatial and Temporal Scheduling}

The Scheduling Code Word (SCW) controls the transmission state and the FIFO selection or antenna selection that the WIFI_CR is to undertake in each timeslot. The SCW is 50 timeslots wide, and is addressed to a specific WIFI_CR by the CR_NMS. It can be transmitted at any time by the CR_NMS, but is initiated only at the beginning of the next 802.11 beacon or GPS timing reset and repeated continually thereafter, starting at each reset, until a new word is received. If $6 \mathrm{Mbps}$ data rates are used, the timeslots are $4 \mathrm{msec}$ long; hence one SCW sequence is executed between the $200 \mathrm{msec} 802.11$ beacon period; or in the case of the GPS synchronization, the word is executed 5 times between the $1000 \mathrm{msec}$ GPS reset period. For data rates of 18 or $54 \mathrm{Mbps}, 2 \mathrm{msec}$ timeslots are used. A maximum of 50 different WIFI_CR terminals forming a CRN can be addressed. More terminals can be addressed by sending alternating SCWs prior to expiry of the current beacon or GPS timing reset interval. Additionally, common quiet periods can be established, allowing a CRN to sense the environment without interference to itself. The number and duration of such periods can be dynamically adjusted.

\section{F. RF and the Sensing Subsystems}

The WIFI_CR's router board has 2 radio cards (Fig 1). One card is configured to work as a data radio operating in diversity mode and is connected to two separate antenna ports: a main transmit/receive port and a receive-only (diversity) port. The diversity RF signal is split with the resulting signals going to the second radio card (which acts as an IEEE 802.11 interference monitor) and another set of stand-alone sensors that are customized for the applications being undertaken. With this configuration the sensor system has continual access to the received spectrum. In the Antenna-centric mode the steerable diversity array can be oriented independently of the primary transmit/receive array allowing the radio to sense the space around itself without affecting the data link's activity.

The greatest source of interference to the WIFI_CR comes from other WiFi radios. The IEEE 802.11 interference monitor card can be set to any channel in the ISM band after which it senses for 500 msecs (between transmission bursts and during receive time slots), recording and quantifying all detectable IEEE $802.11 \mathrm{~g}$ interference seen by WIFI_CR's receive diversity antenna. If the terminal is operating in the Antenna-centric mode with directional arrays, specific sectors can be separately quantified for interference. A 100 channel spectrum analyzer scan over $2400-2500 \mathrm{MHz}$ is taken and a summary of the terminal's own occupancy and transmission history is provided at the same time. The total interference/spectrum occupancy record is returned to the CR_NMS using the format shown in Table I. The CR_NMS can solicit a report from any WIFI_CR terminal in the CRN at any time. Received reports are time stamped by the CR_NMS and placed into a memory called the Radio Environment Awareness Map (REAM) that is accessible to the cognitive engines. It is worthwhile to note that with programming changes to the reporting structure, both at the CR_NMS and WIFI_CR, it is possible to have much faster 


\begin{tabular}{|c|c|c|c|}
\hline \multicolumn{4}{|c|}{ Specific to the addressed WIFI_CR Sensor } \\
\hline \multicolumn{2}{|c|}{ Sensor Attribute } & \multicolumn{2}{|r|}{ Comments } \\
\hline \multicolumn{2}{|c|}{$\begin{array}{l}\text { Sensing Request Message } \\
\text { ID } \\
\text { (S_R_M_ID) }\end{array}$} & \multicolumn{2}{|c|}{$\begin{array}{l}\text { The ID of the CR_NMS sensor inquiry } \\
\text { message that actuated this specific report } \\
\text { message. }\end{array}$} \\
\hline \multicolumn{2}{|c|}{ GPS Time Stamp } & \multicolumn{2}{|c|}{$\begin{array}{l}\text { Time stamp of the SSURF message being } \\
\text { returned to the CR_NMS, taken from the } \\
\text { GPS system just prior to the message being } \\
\text { sent. Null if the WIFI_CR has no GPS }\end{array}$} \\
\hline \multicolumn{2}{|c|}{ GPS Location } & \multicolumn{2}{|c|}{$\begin{array}{l}\text { GPS location. Null if the WIFI_CR has no } \\
\text { GPS }\end{array}$} \\
\hline \multicolumn{2}{|l|}{ Sensor ID } & \multicolumn{2}{|c|}{ Identity of the WIFI_CR (MAC address) } \\
\hline \multicolumn{2}{|c|}{ TX Duty Cycle } & \multicolumn{2}{|c|}{$\begin{array}{l}\text { Ratio of transmission to reception time for } \\
\text { this WIFI_CR }\end{array}$} \\
\hline \multicolumn{2}{|c|}{ TX average data rate } & \multicolumn{2}{|c|}{ Average data rate of link } \\
\hline \multicolumn{2}{|c|}{ Channel Number Sensed } & \multicolumn{2}{|c|}{ Channel (1-11) which the sensor is set on. } \\
\hline \multicolumn{2}{|c|}{$\begin{array}{l}\text { Spectral Occupancy } \\
\text { information }\end{array}$} & \multicolumn{2}{|c|}{$\begin{array}{l}100 \mathrm{MHz} \text { spectrum analysis using an energy } \\
\text { detector with sensitivity of }-85 \mathrm{dBm} / 600 \\
\text { Khz. }\end{array}$} \\
\hline \multicolumn{4}{|c|}{ Specific to each detected IEEE 802.11 interference source (1-n) } \\
\hline \multirow{7}{*}{$\begin{array}{l}\text { Detected } \\
802.11 \mathrm{~g} \\
\text { Interferer } \\
\text { (n) }\end{array}$} & \multicolumn{2}{|l|}{ Channel } & Channel of transmission for (n) \\
\hline & \multicolumn{2}{|l|}{ SSID } & SSID of Interferer (n) \\
\hline & \multicolumn{2}{|l|}{ RSSI } & Average RSSI of all (n) packets \\
\hline & \multicolumn{2}{|c|}{$\begin{array}{l}\text { Number of packets } \\
\text { detected }\end{array}$} & $\begin{array}{l}\text { Number of packets from (n) that } \\
\text { have been detected }\end{array}$ \\
\hline & \multicolumn{2}{|c|}{ Total Duration } & $\begin{array}{l}\text { Sum total duration time of packets } \\
\text { from (n) }\end{array}$ \\
\hline & \multicolumn{2}{|c|}{$\begin{array}{l}\text { MAC source } \\
\text { address }\end{array}$} & MAC source address of (n) \\
\hline & \multicolumn{2}{|c|}{$\begin{array}{l}\text { MAC destination } \\
\text { address }\end{array}$} & MAC dest \\
\hline
\end{tabular}

reporting of shorter messages at intervals in the order of a few to tens of milliseconds.

Sensing of primary users poses a different challenge. The WIFI_CR does not have special detectors for anything other than ISM band interference since each new band and application will have its own requirements. However, the WIFI_CR can provide a received RF signal to an experimental sensor and integrate the sensors output with the EBB (via RS232 data links) so that interference reports can be solicited by the CR_NMS. Experimental TV band, radar, or cellular signal sensors can be tested by such means; additional frequency translation may have to be done at the front end of the WIFI_CR, depending on the application. For such testing the IEEE 802.11 data radio might still be used in order to have a link while proving the integrity of a sensing approach.

\section{G. Cognitive Radio Network Management System}

The Network Management System provides the control interface to the CRN; it acts as a repository for all of the sensed information derived by the network and holds the cognitive algorithms that control the CRN. It acts as the mediating technology between the remotely located cognitive radio terminals and the intelligence embodied by the cognitive engines and REAM. It was designed with several key goals in mind. The first is that it should be easy to modify giving experimenters the ability to configure a $\mathrm{CRN}$ to their requirements. To this extent the CR_NMS is highly modular and uses standard interfaces and protocols to facilitate the use of standard components and libraries: so, for example, the SNMP protocol is used for interaction with wireless nodes and a standard, widelydeployed relational database is used for the REAM. Also, since the intent is to provide the CR_NMS in open source form as an experimental CRN research testbed, open source libraries have been used to facilitate the distribution of the CR_NMS code: for example, the Qt graphical library [9] has been used to implement the CR_NMS graphical user interface.

The CR_NMS architecture consists of software processes that communicate through a message dispatcher having a well defined interface to the modules that control the PHY functions. These modules, which can be replaced or changed, based on the experimenter's needs are the:

- Probe Module: this scans a configured range of IP addresses to detect the availability of a wireless node in a CRN; when a new AP is detected, a new Collector process (described below) is created to handle the CR_NMS interaction with the new AP and its Client stations.

- Collector Module: this undertakes the interaction with an AP and its associated stations including collecting interference information from these nodes, storing this information in a relational database, and setting up various wireless operating parameters on these wireless nodes under the control of the Cognitive Engine or the control of an operator using the CR_NMS Graphical User Interface;

- CR_NMS Graphical User Interface (GUI): this allows an operator to view and control key parameters of wireless nodes in a CRN through a graphical interface; it is implemented using the Qt graphical library to ensure portability and the open source attribute of the CR_NMS.

- External interface for the Cognitive Engine: this provides an API for the cognitive engine to retrieve information about the managed wireless nodes in a CRN, and to control these wireless nodes, through the abstraction layer provided by the CR_NMS;

- Radio Environment Awareness Map (REAM): this relational database, implemented using the PostgreSQL software, stores the sensor information retrieved from the WIFI_CR; the SQL query language provides a flexible way for the cognitive engine to harvest sensing data stored in the REAM database.

\section{H. Cognitive Engines and the REAM}

The construct and operation of the independent and codependent algorithms that form the cognitive engine is the major focus of CR research today; it will likely remain so over the near future as CRNs are deployed. These algorithms interact with the virtual representation of the sensed electromagnetic environment, as embodied by the REAM and it is in such interaction that the learning, correlation, optimization, event recognition, etc. needed to control of the CRN is undertaken.

Application of cognitive engines and REAM may be best illustrated by some CR developments that CRC is undertaking.

- ISM band white space spectrum discovery: A common problem is the selection of a single ISM channel for a distributed set of AP/Client terminals forming long range (1-5 km link) urban roof-top point to multipoint distribution network. Each Client terminal and the AP reports a different set of interference statistics and offered traffic requirements. Both interference and traffic are time variant. This sensed data is stored in the REAM 


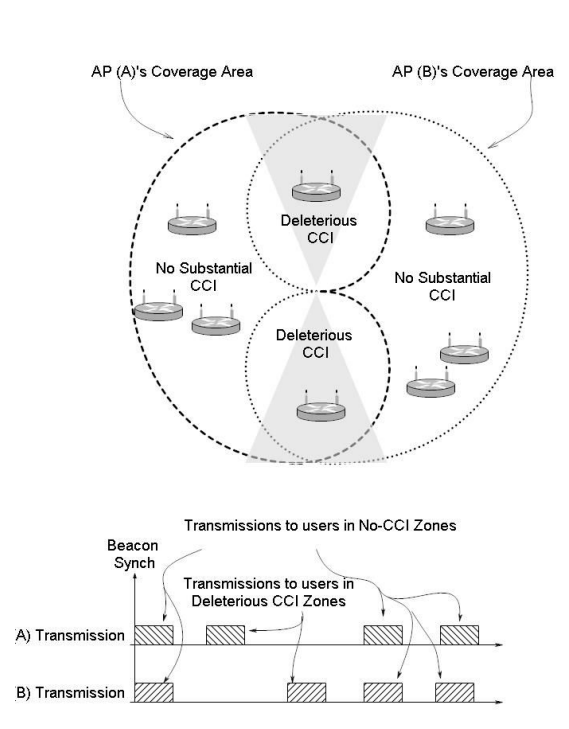

(a) Temporal Isolation

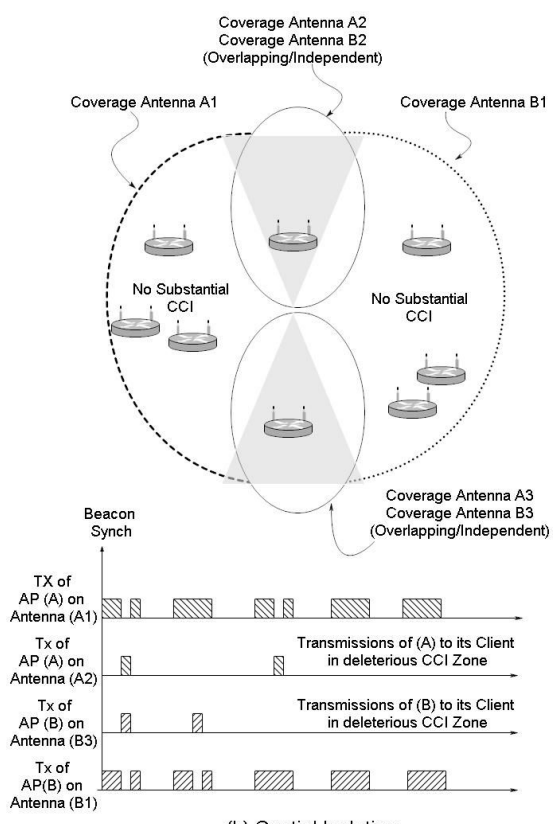

(b) Spatial Isolation

Fig. 3: Single-cell $2 \mathrm{X}$ frequency reuse

which the engine examines to select candidate channels. The algorithm trades fairness against systemic throughput prior to choosing common channel for the network and updates regularly to select switching times in a manner least disruptive to the network [10].

- Policy-based interference control: A CRN is deployed and the terminals within it are asked for location data, provided by GPS. This information is stored and updated in the REAM. A cognitive engine in the CR_NMS queries a third-party database containing deployment locations of intermittent primary users. Interference calculations, supported by propagation statistics are used by the cognitive engine to determine if the locations of the CRN's terminals and the primary user terminals trigger a regulatory requirement. If so, the $\mathrm{CRN}$ switches to an alternative channel identified by the process taken in (a) above.

- Same channel single cell reuse/temporal isolation: Two co-located APs and their associated Clients create a cell of two overlapping same channel sectors. Isolation is provided by directive antennas for each sector. Cochannel interference reports from all terminals are stored in the REAM. A cognitive engine in the CR_NMS, which controls both APs, mines the REAM, identifying users that are suffering excessive co-channel interference. Once identified, IP traffic to these users is scheduled into noninterfering time slots (Fig. 3a) [4].

- Same Channel single cell reuse/spatial isolation: The situation in (c) is repeated. However, now interferenceprone users are assigned antennas/sectors having greater isolation, but narrower coverage (Fig. 3b).

\section{CONCLUSION AND Future WORK}

We have developed a generic cognitive radio by manipulating the operation of an off-the-shelf commodity
IEEE $802.11 \mathrm{~g}$ wireless router using a combined software/hardware overlay. Likely this process can be used with wireless equipment built for other standards; the IEEE 802.16h amendment, for example, has many of the necessary attributes to give IEEE 802.16 (WiMAX) technology a cognitive capability. Having built the WIFI_CR and the CR_NMS (which we call CORAL for cognitive, radio aware, learning platform) we are embarking on a program to test a number of $\mathrm{CR}$ architectures to see if we can achieve both capacity gains and interference resilience while operating in the ISM bands. The work will investigate $600 \mathrm{MHz}$ and $60 \mathrm{GHz} \mathrm{CRN}$ operation in the near future. With the availability of IEEE $802.11 \mathrm{n}$ radios, we will extend many of the concepts alluded to in this paper to MIMO-based CRNs.

\section{REFERENCES}

[1] N. Devroye et al., "Achieved rates in cognitive radio channels," IEEE Transactions on Information Theory, vol. 52, no.5 pp. 1813-1827.

[2] A. Macedo, E. Sousa, "In-cell frequency reuse for broadband indoor wireless systems using sectored antennas," Wireless Personal Cоттиnications, vol. 10, no. 1, June 1999.

[3] P. Mitran et al., "On the capacity and scheduling of a multi-sector cell with co-channel interference knowledge," in Proc. of the 9th IFIP Annual Mediterranean Ad Hoc Workshop, June 2010, France.

[4] Radio Resource Management in Wimax. Vivier, E Editor, John Wiley and Sons, 2009, Coexistence Between 802.16 Systems Operating in Shared Bands Ch.4, pp. 139-186.

[5] C. Stevenson et al., "The first cognitive radio wireless regional area network standard," IEEE Comm. Mag., vol. 47, no.1, pp. 130-138.

[6] R. Patra et al., "WILDNet: design and implementation of high performance WIFI based long distance networks," In Proc. of the 4th USENIX, Symposium on Networked Systems Design Implementation.

[7] D. Panirahi and B. Raman, "TDMA scheduling in long-distance WIFI networks," in Proc. of IEEE INFOCOM 2009, April 2009.

[8] http://madwifi.org

[9] http://qt.nokia.com/

[10] M. Bennai, J. Sydor and M. Rahman, "Automatic channel selection for cognitive radio systems," in Proc. IEEE PIMRC 2010, Istanbul, Turkey. 